\title{
Infrared properties of isolated water ice
}

\author{
P. Ehrenfreund ${ }^{1}$, P.A. Gerakines ${ }^{1,2}$, W.A. Schutte ${ }^{1}$, M.C. van Hemert ${ }^{3}$, and E.F. van Dishoeck $^{1}$ \\ 1 Leiden Observatory, P.O. Box 9513, 2300 RA Leiden, The Netherlands \\ 2 Department of Physics, Rensselaer Polytechnic Institut, Troy, NY 12180-3590, USA \\ ${ }^{3}$ Gorlaeus Laboratories, P.O. Box 9502, 2300, RA Leiden, The Netherlands
}

Received 14 September 1995 / Accepted 18 January 1996

\begin{abstract}
Water ice is the most important component in astrophysical ices and is characterized by a strong broad absorption at $3 \mu \mathrm{m}$, observed in many interstellar spectra. In specific interstellar environments grain mantles might contain only a small amount of water, which is diluted in other volatiles dominant in these regions (e.g. $\mathrm{CO}, \mathrm{O}_{2}, \mathrm{~N}_{2}, \mathrm{CO}_{2}$ ). Theoretical models suggest that interstellar mantles are rich in non-detected molecules such as $\mathrm{O}_{2}$ and $\mathrm{N}_{2}$. If water is diluted in non-polar matrices the water molecules display very sharp discrete bands due to the monomeric and dimeric $\mathrm{H}_{2} \mathrm{O}$. These peculiar bands lie between 2.5 and $2.9 \mu \mathrm{m}$, shortward of the $3 \mu \mathrm{m}$ polymeric $\mathrm{H}_{2} \mathrm{O}$ ice band. We have performed a detailed study of the infrared properties of $\mathrm{H}_{2} \mathrm{O}$ diluted in various concentrations and matrices containing $\mathrm{Ar}, \mathrm{CO}, \mathrm{CO}_{2}, \mathrm{O}_{2}, \mathrm{~N}_{2}$ and naphthalene. Each molecule present in the matrix has its individual fingerprint in the spectrum of isolated $\mathrm{H}_{2} \mathrm{O}$ and can be traced by the resulting band position of the individual $\mathrm{H}_{2} \mathrm{O}$ molecules and overall pattern of the infrared spectrum. The concentration of $\mathrm{H}_{2} \mathrm{O}$ in the matrix determines the specific intensity ratios of the monomeric, dimeric and polymeric water bands. We discuss the possible detection of isolated water on low temperature and ultraviolet shielded grain mantles covered with non-polar ices. Together with astronomical spectra taken by the ISO satellite these laboratory data will be extremely valuable for the determination of the grain mantle composition in dense clouds. Due to the unique spectral signature of $\mathrm{H}_{2} \mathrm{O} / \mathrm{CO} / \mathrm{O}_{2}$ mixtures the detection of isolated water may allow us to infer the presence and abundance of solid $\mathrm{O}_{2}$.
\end{abstract}

Key words: ISM: molecules - ISM: abundances; dust - methods: laboratory

\section{Introduction}

Icy grain mantles are dust components which are formed in dense interstellar clouds (Greenberg 1982, d'Hendecourt et al. 1986). Many infrared observations towards obscured sources

Send offprint requests to: $\mathrm{P}$. Ehrenfreund have been performed to determine the molecules residing on interstellar grains (e.g. Willner et al. 1982, d'Hendecourt \& de Muizon 1989, Grim et al. 1991). These observations imply that the grain mantle constituents are either directly accreted from the gas phase or are produced by surface reactions. Energetic ultraviolet photoprocessing of icy grain mantles enables the formation of new molecules and radicals (d'Hendecourt et al. 1985). Grain surface reactions play an active role in interstellar chemistry and strongly influence gas phase abundances. Desorption processes return molecules, which were altered on the grains, back into the gas phase (Greenberg 1976, Léger et al. 1985, Schutte \& Greenberg 1991, Hasegawa \& Herbst 1993). The infrared properties of astrophysically relevant ice mixtures have been studied extensively in the laboratory (see Tielens 1991 for a review). Comparison of these laboratory data with interstellar observations have led to the detection of many interstellar solid state molecules (see reviews by Whittet 1993, Schmitt 1994, Schutte 1996).

Water ice is the most abundant molecule in grain mantles. The infrared spectrum of water ice is dominated by a strong band at $3.08 \mu \mathrm{m}\left(3250 \mathrm{~cm}^{-1}\right)$, due to the $\mathrm{O}-\mathrm{H}$ stretching vibration of $\mathrm{H}_{2} \mathrm{O}$ and a weaker absorption centred at $6 \mu \mathrm{m}\left(1660 \mathrm{~cm}^{-1}\right)$, associated with the bending mode of $\mathrm{H}_{2} \mathrm{O}$ ice. The strong broad absorption of water at $3 \mu \mathrm{m}$ has been observed toward more than 100 sources (see Schmitt 1994 for a review). Calculations indicate that the growth of ice mantles cannot result from $\mathrm{H}_{2} \mathrm{O}$ accretion from the gas phase and only surface reactions produce $\mathrm{H}_{2} \mathrm{O}$ ice on grain mantles in sufficient abundances (e.g. Whittet, 1993). However, water ice formation requires high densities and ultraviolet shielding. For example, the spectra of the diffuse medium towards the galactic center sources IRS 7 and IRS 19 exhibit weak water ice features, compared to dense clouds (Sandford et al. 1991, Pendleton et al. 1994). Studies of the $3 \mu \mathrm{m}$ absorption profile indicate the influence of different physical environments on the structure and purity of water ice. Laboratory studies show that infrared features of $\mathrm{H}_{2} \mathrm{O}$ ice display reversible and irreversible temperature effects in band shape and position (Hagen et al. 1981). Therefore, the shape of water ice can be regarded as a good indicator of the physical state and the thermal evolution of interstellar ices (van der Bult et al. 1985, Smith et 
al. 1989). In the spectra of the late-type OH/IR star OH 231.8 +4.2 the observations are fit best with pure water ice, whereas a "shoulder" between 3.2 and $3.6 \mu \mathrm{m}$ is observed in molecular clouds. This absorption is probably due to other molecules contained within the grain mantles (Whittet et al. 1993).

Recent work indicates layered structures on grain mantles and the existence of polar and non-polar ices (Sandford et al. 1988, Whittet \& Duley 1991, Tielens et al. 1991). Laboratory studies of the shape and peak position of the solid CO band in astrophysically relevant ice mixtures show a two-component structure of solid $\mathrm{CO}$ at $2140 \mathrm{~cm}^{-1}$. A narrow $\mathrm{CO}$ band is observed in mixtures dominated by non-polar molecules $(\mathrm{CO}$, $\mathrm{CO}_{2}, \mathrm{O}_{2}, \mathrm{~N}_{2}$ ), whereas a broad component originates in polar mixtures, such as $\mathrm{H}_{2} \mathrm{O}$ ice. Observations show that many lines of sight contain (at least) two independent grain mantle components (Tielens et al. 1991). At low densities, atomic $\mathrm{H}$ is an important constituent in the gas phase and dominates in grain surface reactions forming mostly $\mathrm{H}_{2} \mathrm{O}$-rich ices (including $\mathrm{CO}$, $\mathrm{NH}_{3}$ and $\mathrm{CH}_{4}$ ). At high densities, where heavy species are much more abundant than atomic $\mathrm{H}$, grains might accrete a mantle of non-polar ices dominated by $\mathrm{CO}, \mathrm{CO}_{2}, \mathrm{~N}_{2}$ and $\mathrm{O}_{2}$. However, non-polar ices are much more volatile than solid $\mathrm{H}_{2} \mathrm{O}$ and will be evaporated around luminous protostars. Thus the line of sight towards an embedded object may be dominated by grains with different mantle components: $\mathrm{H}_{2} \mathrm{O}$-rich ices close to the star and non-polar ices far away. The existence of an"onion-like" structure has been suggested by observations and laboratory studies of solid $\mathrm{CO}$ and provide strong constraints for the evolution and life cycle of grains in interstellar clouds (Whittet \& Duley 1991, Tielens et al. 1991).

Theoretical models demonstrate that in high density environments where grains accrete mainly non-polar gas phase molecules $\left(\mathrm{CO}, \mathrm{N}_{2}, \mathrm{O}_{2}\right.$ ), a non-negligible quantity of $\mathrm{H}_{2} \mathrm{O}$ may still co-condense (Tielens \& Hagen 1982, d'Hendecourt et al. 1985). When $\mathrm{H}_{2} \mathrm{O}$ molecules are isolated they do not interact with each other and sharp discrete bands due to monomeric $\mathrm{H}_{2} \mathrm{O}$ (one single $\mathrm{H}_{2} \mathrm{O}$ molecule surrounded by other molecules) and dimeric $\mathrm{H}_{2} \mathrm{O}$ can be observed around $2.5 \mu \mathrm{m}$. The specific position and shape of these bands is determined by the interaction with adjacent molecules in the ice (Hagen \& Tielens 1981). The described bands of isolated water fall shortward of the 3 $\mu \mathrm{m}$ band of polymeric water ice and can only be observed by satellite observations due to strong telluric absorption of water vapour in this wavelength region.

The molecules $\mathrm{O}_{2}$ and $\mathrm{N}_{2}$ might be abundant grain mantle constituents in non-polar ices. $\mathrm{O}_{2}$ and $\mathrm{N}_{2}$ are homonuclear diatomic molecules. Their vibration and rotation do not produce a change in dipole moment making them infrared inactive and unobservable at radio wavelengths. Interaction with adjacent molecules in the solid state can break the symmetry of the vibrations and modes become weakly IR active. The $\mathrm{O}=\mathrm{O}$ fundamental vibration was detected in a $\mathrm{CO}_{2}$ matrix at 1550 $\mathrm{cm}^{-1}$ (Ehrenfreund et al. 1992) and will be searched with ISO, the Infrared Space Observatory. Recent observations towards VS17 showed that the CO profile at $4.67 \mu \mathrm{m}$ is best fit by a $\mathrm{CO} / \mathrm{O}_{2}$ mixture (Kerr et al. 1993, Tielens et al. 1991). $\mathrm{N}_{2}$ shows its vibrational transition at $2330 \mathrm{~cm}^{-1}$ and will be difficult to detect, because the very strong asymmetric stretch of $\mathrm{CO}_{2}$ and satellite bands of $\mathrm{CO}_{2}$ are located very close to this position (at $2340 / 2330 \mathrm{~cm}^{-1}$ ). Indirect methods to infer the presence of homonuclear molecules are (i) to study the profile of the $\mathrm{CO}$ and $\mathrm{CO}_{2}$ bands (Tielens et al. 1991, Ehrenfreund et al. 1996) and (ii) the band position of isolated water features.

The infrared spectrum of $\mathrm{H}_{2} \mathrm{O}$ in low temperature matrices has been the subject of many investigations (Benedict et al. 1956, Mann et al. 1974, Barnes et al. 1980, Fredin et al. 1977, Murby et al. 1979, Hagen \& Tielens 1981, Tso \& Lee 1985). Isolated water was previously studied in various matrices in order to determine hydrogen-bonding and dimeric structure (van Thiel et al. 1957). Monomeric water exhibits a complex spectrum in noble gas matrices due to rotation and nuclear spin conversion. Rotation does not occur in matrices such as $\mathrm{N}_{2}$ or $\mathrm{CO}$ (Barnes et al. 1980). The role of water isolated in $\mathrm{CO}$ has been investigated by Hagen \& Tielens (1981), who studied the evolution of the spectrum upon variation of concentration and during warm-up. These authors also summarize earlier matrix work on isolated water in $\mathrm{Ar}$ and $\mathrm{N}_{2}$ in combination with gas phase data and claim a bifurcated structure for the water dimer in solid $\mathrm{CO}$. The role of hydrogen bonding of $\mathrm{H}_{2} \mathrm{O}$ in solid $\mathrm{O}_{2}$ has been studied by Tso \& Lee (1985).

In a non-polar ice mixture on interstellar grains the water molecules will predominantly be surrounded by molecules such as $\mathrm{CO}, \mathrm{O}_{2}, \mathrm{~N}_{2}$ and $\mathrm{CO}_{2}$. Possibly polycyclic aromatic hydrocarbons (PAHs), which are thought to be ubiquitous in the interstellar medium (Puget \& Léger 1989) could be abundant ice constituents as well. Appreciable quantities of $\mathrm{CO}_{2}$ will be present only when the grain mantles have been exposed to ultraviolet irradiation (Grim \& d'Hendecourt 1986, d'Hendecourt et al. 1986). In this paper we present a study of isolated water in astrophysically relevant non-polar ice mixtures. We studied the infrared properties of water ice diluted in matrices containing $\mathrm{CO}, \mathrm{N}_{2}, \mathrm{O}_{2}, \mathrm{CO}_{2}$ and naphthalene $\left(\mathrm{C}_{10} \mathrm{H}_{8}\right)$. The changes of the spectra during warm-up and UV photolysis were monitored. The band strength was measured in various matrices and as a function of temperature and UV processing in order to define the environmental conditions where water ice in this form could be successfully detected in space. The presented results are a part of the program of the Leiden Observatory dedicated to the solid state data base for ISO.

\section{Experimental}

In order to produce interstellar analogs in the laboratory a pure gas or gas mixture was condensed in a high vacuum chamber on the surface of a CsI window, cooled by a closed cycle $\mathrm{He}$ refrigerator to $10 \mathrm{~K}$. UV irradiation was performed by a microwave excited hydrogen flow lamp. The output of this source has a sharp emission peak at $121.6 \mathrm{~nm}($ Lyman $\alpha)$ and additional bands centred at $1360,1450,1600$ and $2800 \AA$, which produce a flux of approximately $10^{15}$ photons $\mathrm{cm}^{-2} \mathrm{~s}^{-1}$ (Weber \& Greenberg 1985). Infrared transmission spectra were obtained with a BioRad FTS 40A spectrometer at a resolution of $1 \mathrm{~cm}^{-1}$.

\section{(c) European Southern Observatory - Provided by the NASA Astrophysics Data System}


Gases and gas mixtures have been prepared in a glass vacuum manifold. The $\mathrm{H}_{2} \mathrm{O}$ vapor is obtained from triply distilled demineralized water purified by three freeze thaw cycles carried out under vacuum. The purity of the used gases are: $\mathrm{CO}(99.9997$ \% Messer Griesheim), $\mathrm{CO}_{2}$ (99.9997 \% Messer Griesheim), $\mathrm{N}_{2}$ (99.9997 \% Messer Griesheim) and $\mathrm{O}_{2}(99.9997 \%$ Messer Griesheim). Naphthalene vapor was obtained from a solid sample ( $99.9 \%$ purity).

The deposition rate and sample thickness growth rate were about $10^{15}$ molec $\mathrm{cm}^{-2} \mathrm{~s}^{-1}$ and $1 \mu \mathrm{m} \mathrm{hr}^{-1}$, respectively. Deposition times in the order of 5-10 min resulted in a sample thickness of approximately $0.1 \mu \mathrm{m}$. A detailed description of the experimental setup has been given by Gerakines et al. (1995). Experiments of water ice isolated in naphthalene were performed such that naphthalene and $\mathrm{H}_{2} \mathrm{O}$ were deposited through seperate deposition tubes. This procedure was required due to the very low vapor pressure of naphthalene at room temperature $(\sim 0.2$ mbar).

Water accretion on the substrate (CsI window) during the experimental phase due to imperfect vacuum conditions has been corrected in all displayed spectra (see also Gerakines et al. 1995).

\section{Results}

Tables 1-4 summarize the results of our experiments. The IR spectrum of isolated water displays a set of bands due to stretching and bending vibrations of monomers, dimers, trimers and higher multimers resulting from the interaction with the surrounding molecules. Assignments of the monomeric and dimeric absorptions to specific vibrational modes in this paper are based on a comparison with gas phase data and earlier matrix work. We do not discuss weak multimeric features, since their assignment is rather speculative. Symbols $\nu_{1}, \nu_{2}$, and $\nu_{3}$ refer to the symmetric stretching mode, the bending mode, and the asymmetric stretching mode of $\mathrm{OH}$, respectively. The first band in the stretching mode vibration at high frequencies is due to the asymmetric $\left(\nu_{3}\right)$ stretch of the $\mathrm{H}_{2} \mathrm{O}$ monomer followed by a pattern of bands which depends strongly on the matrix.

The asymmetric stretch of the $\mathrm{H}_{2} \mathrm{O}$ monomer in ices is always red-shifted relative to the gas phase value of $\mathrm{H}_{2} \mathrm{O}$ at 3755.6 $\mathrm{cm}^{-1}$ and stronger shifts appear when the polarizability of the matrix increases. The band positions of the dimers depend on their structure in the matrix and show a different pattern according to the molecular environment (Hagen \& Tielens 1981). The plausible configurations of water dimers are shown in Fig. 1. Infrared spectra of $\mathrm{H}_{2} \mathrm{O}$ dimers are characterized by two sets of absorption peaks. We assign in this paper " $\mathrm{A}$ " as the $\mathrm{e}^{-}$ acceptor (H-atom) of one $\mathrm{H}_{2} \mathrm{O}$ molecule and " $\mathrm{eD}$ " as the $\mathrm{e}^{-}$ donor (O-atom) of the other $\mathrm{H}_{2} \mathrm{O}$ molecule in a dimer. The $\mathrm{H}_{2} \mathrm{O}$ dimeric structure in matrices such as $\mathrm{Ar}$ or $\mathrm{N}_{2}$ is linear, whereas the structure of dimers in $\mathrm{CO}$ is bifurcated (Morokuma \& Pedersen 1968, Barnes et al. 1980, Hagen \& Tielens 1981). The infrared spectroscopic pattern shows a characteristic band order and spacing of the $\nu_{3}, \nu_{2}$ and $\nu_{1}$ monomers and dimers, reflecting interactions within the matrix. We show in Fig. 2 two

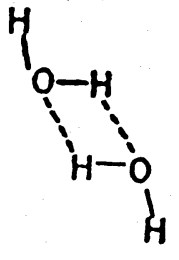

\section{cyclic \\ bifurcated \\ linear}

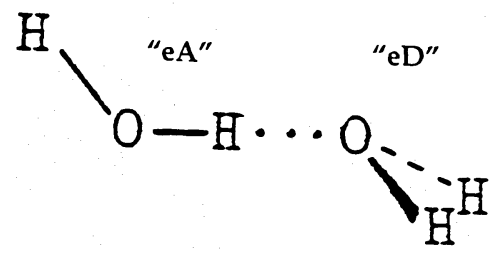

Fig. 1. Possible configurations of $\mathrm{H}_{2} \mathrm{O}$ dimers (Morokuma \& Pedersen, 1968). "eA" is assigned as the $\mathrm{e}^{-}$acceptor (H-atom) and " $\mathrm{eD}$ " as the $\mathrm{e}^{-}$donor (O-atom) of the hydrogen-bonding pair.

spectra representative of the linear (in $\mathrm{N}_{2}$ matrix) and bifurcated (in $\mathrm{CO}$ matrix) configuration of the $\mathrm{H}_{2} \mathrm{O}$ dimer. We also indicated the components of the spectral pattern in the stretching mode region. For a detailed description about identification, frequency shifts, bending mode region and relative intensities of the $\mathrm{OH}$ modes of dimeric $\mathrm{H}_{2} \mathrm{O}$ we refer to Hagen \& Tielens (1981).

\subsection{Isolated water in solid $\mathrm{N}_{2}$ and $\mathrm{O}_{2}$}

In Fig. 3 and 4 we present the infrared spectra of $\mathrm{H}_{2} \mathrm{O}$ in matrices containing $\mathrm{O}_{2}$ and $\mathrm{N}_{2}$. The spectra show two distinct regions from $3800-3000 \mathrm{~cm}^{-1}$ and $1700-1500 \mathrm{~cm}^{-1}$, corresponding to the stretching and bending vibrations of water ice, respectively.

In Fig. 3 the absorber to matrix ratio is about 1:10 and a significant fraction of water ice is still in its polymeric form, which is observed as a broad underlying feature between 3600 $3200 \mathrm{~cm}^{-1}$. Bands which are superimposed on this broad feature correspond to water multimers (e.g. tetramers and higher multimers). The sharp bands at higher frequency preceding the broad polymeric feature are due to monomers, dimers and small multimers of water ice, as specified in the figure caption and Table 1. Monomeric and dimeric bands are rather weak in a matrix where the absorber to matrix ratio is about $1: 10$ and the strongest bands in all spectra correspond to water ice multimers. Also in the bending mode region (Fig. 3b) polymeric water shows a dominant broad feature around $1630 \mathrm{~cm}^{-1}$. For comparison in Fig. 4 where a dilution of 1:100 is used, the broad underlying polymeric water ice feature has largely disappeared. In the stretching mode region the first sharp band in all spectra toward high frequency is due to the monomeric asymmetric $\mathrm{OH}$ stretching mode $\left(\nu_{3}\right)$ of water ice.

The assignments of all bands for the stretching and bending mode region due to monomeric and dimeric water ice are

\section{(c) European Southern Observatory - Provided by the NASA Astrophysics Data System}


Table 1. Comparison of the position (in $\mathrm{cm}^{-1}$ ) of IR absorption features of isolated water ice in various matrices. Symbols $\nu_{1}, \nu_{2}$, and $\nu_{3}$ refer to the symmetric stretching mode, the bending mode, and the asymmetric OH stretching mode, respectively. "eA" and "eD" are as defined in Fig. 1. The corresponding $\mathrm{H}_{2} \mathrm{O}$ gas phase values are located at $3755.59 \mathrm{~cm}^{-1}\left(\nu_{3}\right), 3656.65 \mathrm{~cm}^{-1}\left(\nu_{1}\right)$ and $1594.59 \mathrm{~cm}^{-1}\left(\nu_{2}\right)(B e n e d i c t$ et al. 1956). (b) denotes blend. Bands which could not be detected are indicated by "-".

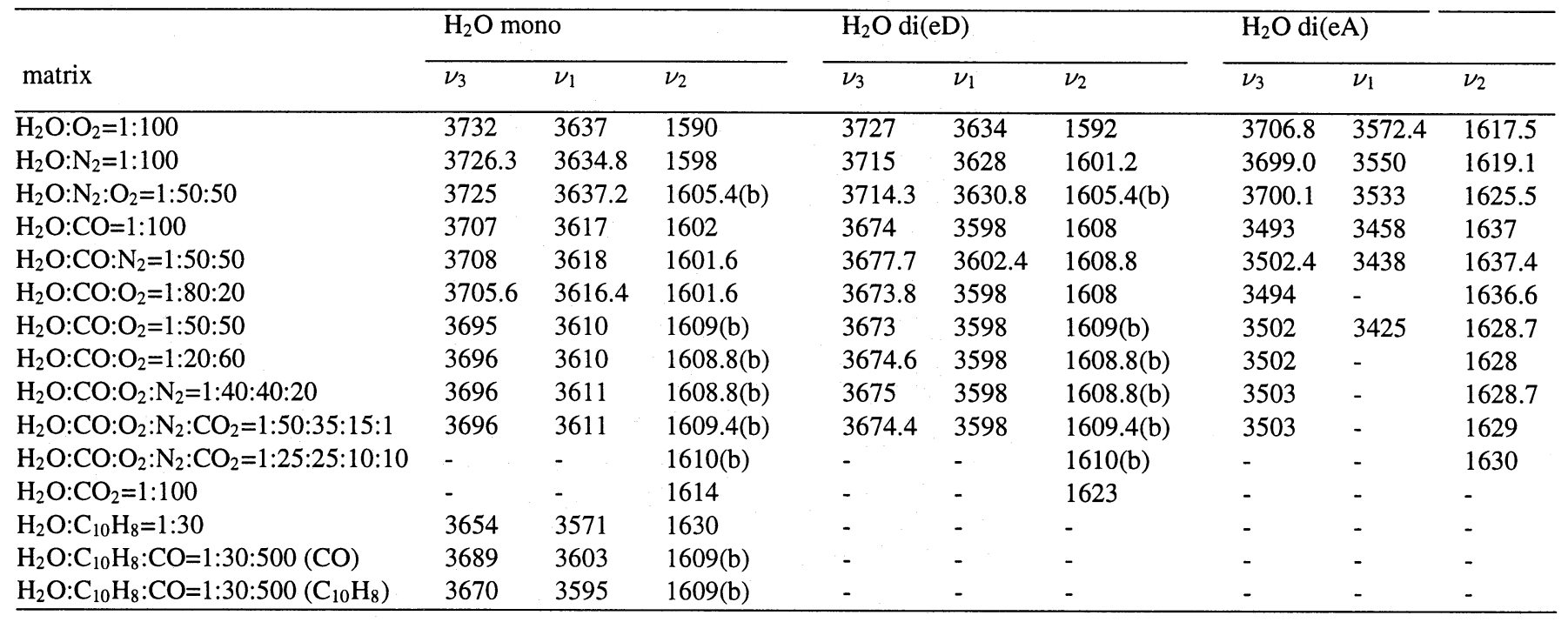

Table 2. Band width (FWHM in $\mathrm{cm}^{-1}$ ) of IR absorption features of isolated water ice in various matrices. Symbols $\nu_{1}, \nu_{2}$, and $\nu_{3}$ refer to the symmetric stretching mode, the bending mode, and the asymmetric OH stretching mode, respectively. "eA" and "eD" are as defined in Fig. 1. "-" denotes that the bands are either too weak or strongly blended to achieve a valuable band width measurement.

\begin{tabular}{|c|c|c|c|c|c|c|c|c|c|}
\hline \multirow[b]{2}{*}{ matrix } & \multicolumn{3}{|c|}{$\mathrm{H}_{2} \mathrm{O}$ mono } & \multicolumn{3}{|c|}{$\mathrm{H}_{2} \mathrm{O} \operatorname{di}(\mathrm{eD})$} & \multicolumn{3}{|c|}{$\mathrm{H}_{2} \mathrm{O} \operatorname{di}(\mathrm{eA})$} \\
\hline & $\nu_{3}$ & $\nu_{1}$ & $\nu_{2}$ & $\nu_{3}$ & $\nu_{1}$ & $\nu_{2}$ & $\nu_{3}$ & $\nu_{1}$ & $\nu_{2}$ \\
\hline $\mathrm{H}_{2} \mathrm{O}: \mathrm{O}_{2}=1: 100$ & 6 & - & 2 & 4.3 & - & 2.5 & 6 & 5 & 4 \\
\hline $\mathrm{H}_{2} \mathrm{O}: \mathrm{N}_{2}=1: 100$ & 3.3 & 2 & 2.5 & 5 & 2.5 & 4 & 6 & 6 & 3.2 \\
\hline $\mathrm{H}_{2} \mathrm{O}: \mathrm{N}_{2}: \mathrm{O}_{2}=1: 50: 50$ & 6.4 & 7 & $6.7(b)$ & 8.7 & 5 & $6.7(b)$ & 7.2 & 18 & 14 \\
\hline $\mathrm{H}_{2} \mathrm{O}: \mathrm{CO}=1: 100$ & 3.4 & 2 & 2 & 5 & 3 & 3 & 7 & - & 3 \\
\hline $\mathrm{H}_{2} \mathrm{O}: \mathrm{CO}: \mathrm{N}_{2}=1: 50: 50$ & 4.4 & 3.5 & 4 & 15 & 9 & 5.2 & 24 & - & 9 \\
\hline $\mathrm{H}_{2} \mathrm{O}: \mathrm{CO}: \mathrm{O}_{2}=1: 80: 20$ & 5.6 & 5 & 4.4 & 10 & 8 & 5.5 & 10 & - & - \\
\hline $\mathrm{H}_{2} \mathrm{O}: \mathrm{CO}: \mathrm{O}_{2}=1: 50: 50$ & 9.6 & 7.6 & $9(\mathrm{~b})$ & 16 & 10 & $9(b)$ & 28 & - & 13 \\
\hline $\mathrm{H}_{2} \mathrm{O}: \mathrm{CO}: \mathrm{O}_{2}=1: 20: 60$ & 10 & 7.8 & $10(b)$ & 16 & 12 & $10(\mathrm{~b})$ & 30 & - & 13 \\
\hline $\mathrm{H}_{2} \mathrm{O}: \mathrm{CO}: \mathrm{O}_{2}: \mathrm{N}_{2}=1: 40: 40: 20$ & 12 & 9 & $11(b)$ & 19 & 12 & $11(b)$ & 33 & - & 15 \\
\hline $\mathrm{H}_{2} \mathrm{O}: \mathrm{CO}: \mathrm{O}_{2}: \mathrm{N}_{2}: \mathrm{CO}_{2}=1: 50: 35: 15: 1$ & 10.3 & 8.4 & $8.9(b)$ & 13 & - & 8.9 (b) & 30 & - & 10 \\
\hline $\mathrm{H}_{2} \mathrm{O}: \mathrm{CO}_{2}=1: 100$ & - & - & 4 & - & - & - & - & - & - \\
\hline $\mathrm{H}_{2} \mathrm{O}: \mathrm{C}_{10} \mathrm{H}_{8}=1: 30$ & 48 & 44 & 7 & - & - & - & - & - & - \\
\hline
\end{tabular}

listed in Table 1. Additional weak features below $3400 \mathrm{~cm}^{-1}$ are of multimeric origin. The assignment of higher multimers is ambiguous and prevents a clear identification. Table 2 specifies the band width (FWHM) of isolated water features. The bands are narrow in mixtures containing only $\mathrm{O}_{2}$ and $\mathrm{N}_{2}$, but the band width increases in a matrix containing both molecular oxygen and nitrogen. It must be noted that the position and width due to water ice monomers, dimers and multimers do not vary with $\mathrm{H}_{2} \mathrm{O}$ concentration, as long as the matrix composition stays the same.
Table 3 summarizes the band ratios of the main infrared features normalized to the $\nu_{3}$ band of the monomer in each individual mixture. It can be seen that in an $\mathrm{O}_{2}$ matrix the ratio of dimers (and trimers) to monomers is much higher than in other matrices. In Table 4 we compare the band ratios in mixtures with different concentration. At low dilution the monomer and dimer absorption of $\mathrm{H}_{2} \mathrm{O}$ are weak compared to multimeric features. At an absorber to matrix ratio of 1:10 the ratio of dimers and multimers to monomers increases much stronger for the multi- 
Table 3. Band depth ratio of IR absorption features of isolated water ice in various matrices. Symbols $\nu_{1}, \nu_{2}$, and $\nu_{3}$ refer to the symmetric stretching mode, the bending mode, and the asymmetric $\mathrm{OH}$ stretching mode, respectively. "eA" and "eD" are as defined in Fig. 1. All bands are normalized to the $\nu_{3}$ monomer. "-" denotes that the bands are either too weak or strongly blended.

\begin{tabular}{|c|c|c|c|c|c|c|c|c|c|}
\hline \multirow[b]{2}{*}{ matrix } & \multicolumn{3}{|c|}{$\mathrm{H}_{2} \mathrm{O}$ mono } & \multicolumn{3}{|c|}{$\mathrm{H}_{2} \mathrm{O}$ di(eD) } & \multicolumn{3}{|c|}{$\mathrm{H}_{2} \mathrm{O}$ di(eA) } \\
\hline & $\nu_{3}$ & $\nu_{1}$ & $\nu_{2}$ & $\nu_{3}$ & $\nu_{1}$ & $\nu_{2}$ & $\nu_{3}$ & $\nu_{1}$ & $\nu_{2}$ \\
\hline $\mathrm{H}_{2} \mathrm{O}: \mathrm{O}_{2}=1: 100$ & 1 & - & 0.43 & 0.77 & - & 0.71 & 0.82 & 0.67 & 0.86 \\
\hline $\mathrm{H}_{2} \mathrm{O}: \mathrm{N}_{2}=1: 100$ & 1 & 0.19 & 0.83 & 0.26 & 0.06 & 0.43 & 0.26 & 0.21 & 0.15 \\
\hline $\mathrm{H}_{2} \mathrm{O}: \mathrm{N}_{2}: \mathrm{O}_{2}=1: 50: 50$ & 1 & 0.154 & 0.86 & 0.28 & 0.06 & - & 0.34 & 0.28 & 0.24 \\
\hline $\mathrm{H}_{2} \mathrm{O}: \mathrm{CO}=1: 100$ & 1 & 0.26 & 0.63 & 0.42 & 0.078 & 0.15 & 0.22 & - & 0.045 \\
\hline $\mathrm{H}_{2} \mathrm{O}: \mathrm{CO}: \mathrm{N}_{2}=1: 50: 50$ & 1 & 0.23 & 0.59 & 0.35 & 0.042 & 0.18 & 0.16 & - & 0.083 \\
\hline $\mathrm{H}_{2} \mathrm{O}: \mathrm{CO}: \mathrm{O}_{2}=1: 80: 20$ & 1 & 0.22 & 0.6 & 0.42 & 0.06 & 0.24 & 0.24 & - & 0.09 \\
\hline $\mathrm{H}_{2} \mathrm{O}: \mathrm{CO}: \mathrm{O}_{2}=1: 50: 50$ & 1 & 0.26 & 0.6 & 0.69 & 0.095 & - & 0.34 & - & 0.22 \\
\hline $\mathrm{H}_{2} \mathrm{O}: \mathrm{CO}: \mathrm{O}_{2}=1: 20: 60$ & 1 & 0.23 & 0.63 & 0.79 & 0.08 & - & 0.36 & - & 0.23 \\
\hline $\mathrm{H}_{2} \mathrm{O}: \mathrm{CO}: \mathrm{O}_{2}: \mathrm{N}_{2}=1: 40: 40: 20$ & 1 & 0.25 & 0.71 & 0.95 & 0.11 & - & 0.46 & - & 0.3 \\
\hline $\mathrm{H}_{2} \mathrm{O}: \mathrm{CO}: \mathrm{O}_{2}: \mathrm{N}_{2}: \mathrm{CO}_{2}=1: 50: 35: 15: 1$ & 1 & 0.28 & 0.625 & 0.53 & - & - & 0.24 & - & 0.12 \\
\hline $\mathrm{H}_{2} \mathrm{O}: \mathrm{C}_{10} \mathrm{H}_{8}=1: 30$ & 1 & 0.5 & 0.35 & - & - & - & - & - & - \\
\hline
\end{tabular}

Table 4. Comparison of the band depth ratio of IR absorption features of isolated water ice in mixtures of dilution 1:100 and 1:10. Symbols $\nu_{2}$, and $\nu_{3}$ refer to the bending mode and the asymmetric $\mathrm{OH}$ stretching mode, respectively. "eA" and "eD" are as defined in Fig. 1. (b) denotes blend. All bands are normalized to the $\nu_{3}$ monomer. Polymers - denoted by "po" - are the dominant features in mixtures of absorber to matrix ratio of $1: 10$ and weak or absent in mixtures of $1: 100$.

\begin{tabular}{|c|c|c|c|c|c|c|c|c|}
\hline \multirow[b]{2}{*}{ matrix } & \multicolumn{2}{|c|}{$\mathrm{H}_{2} \mathrm{O}$ mono } & \multicolumn{2}{|c|}{$\mathrm{H}_{2} \mathrm{O}$ di(eD) } & \multicolumn{2}{|c|}{$\mathrm{H}_{2} \mathrm{O}$ di $(\mathrm{eA})$} & \multicolumn{2}{|l|}{$\mathrm{H}_{2} \mathrm{O}$ po } \\
\hline & $\nu_{3}$ & $\nu_{2}$ & $\nu_{3}$ & $\nu_{2}$ & $\nu_{3}$ & $\nu_{2}$ & po $\left(3690 \mathrm{~cm}^{-1}\right)$ & po $\left(1630 \mathrm{~cm}^{-1}\right)$ \\
\hline$\overline{\mathrm{H}_{2} \mathrm{O}: \mathrm{O}_{2}=1: 100}$ & 1 & 0.43 & 0.77 & 0.71 & 0.82 & 0.86 & 0.77 & - \\
\hline $\mathrm{H}_{2} \mathrm{O}: \mathrm{O}_{2}=1: 10$ & 1 & 0.55 & 1 & 0.70 & 2.77 & 1.96 & 3.7 & 1.85 \\
\hline $\mathrm{H}_{2} \mathrm{O}: \mathrm{N}_{2}=1: 100$ & 1 & 0.83 & 0.26 & 0.43 & 0.26 & 0.15 & 0.41 & - \\
\hline $\mathrm{H}_{2} \mathrm{O}: \mathrm{N}_{2}=1: 10$ & 1 & 0.51 & 0.59 & 0.62 & $\mathrm{~b}$ & $\mathrm{~b}$ & 3.24 & 0.84 \\
\hline $\mathrm{H}_{2} \mathrm{O}: \mathrm{N}_{2}: \mathrm{O}_{2}=1: 50: 50$ & 1 & 0.86 & 0.28 & $\mathrm{~b}$ & 0.34 & 0.24 & 0.35 & - \\
\hline $\mathrm{H}_{2} \mathrm{O}: \mathrm{N}_{2}: \mathrm{O}_{2}=1: 5: 5$ & 1 & $\mathrm{~b}$ & 1.18 & 1.5 & $\mathrm{~b}$ & $\mathrm{~b}$ & 5.7 & 1.53 \\
\hline
\end{tabular}

component mixture $\mathrm{H}_{2} \mathrm{O} / \mathrm{N}_{2} / \mathrm{O}_{2}$, compared to simple matrices containing either $\mathrm{O}_{2}$ or $\mathrm{N}_{2}$.

\subsection{Isolated water in $\mathrm{CO} / \mathrm{O}_{2} / \mathrm{N}_{2}$ matrices}

$\mathrm{CO}$ is the most abundant ice component after water in the interstellar medium and should be the main constituent of non-polar ices. CO has been observed towards many sources (Tielens et al. 1991) and in particular in the Serpens and Taurus Dark Cloud systems (Chiar et al. 1994, 1995). In the Serpens cloud a mean $\mathrm{CO}$ abundance in dust of $40 \%$ of the $\mathrm{H}_{2} \mathrm{O}$ abundance was observed, while in Taurus $\mathrm{CO}$ has a $25 \%$ abundance. The high resolution study of $\mathrm{CO}$ profiles in these regions indicates the presence of two distinct phases of $\mathrm{CO}$ bearing ice, one dominated by pure $\mathrm{CO}$ or $\mathrm{CO}$ mixed with non-polar molecules, the other with $\mathrm{H}_{2} \mathrm{O}$ as the primary constituent (Chiar et al. 1994, 1995).

Fig. 5 shows the behaviour of isolated water in matrices containing $\mathrm{CO}, \mathrm{O}_{2}$ and $\mathrm{N}_{2}$. Band assignments are listed in Table 1. The behaviour and profile of the $\mathrm{CO}$ band in these mixtures will be presented in a forthcoming paper (Ehrenfreund et al. 1996).
Comparison of the spectra shows that the $\mathrm{CO}$ molecule dominates the interaction within the matrix, even in the presence of $\mathrm{N}_{2}$ or small amounts of $\mathrm{O}_{2}$. The bifurcated structure of the $\mathrm{H}_{2} \mathrm{O}$ dimer in CO, defined by Hagen \& Tielens (1981), is retained in all mixtures containing $\mathrm{CO}$. However, the presence of equal amounts of $\mathrm{CO}$ and $\mathrm{O}_{2}$ induces large changes in band position indicative of a strong matrix perturbation. This perturbation can also be seen in the profile and width of the CO band at 2140 $\mathrm{cm}^{-1}$ (Ehrenfreund et al. 1996).

Our results indicate that the simultaneous presence of $\mathrm{CO}$ and $\mathrm{O}_{2}$ in the matrix induces a strong interaction with the water molecules, resulting in a large red-shift of the $\mathrm{H}_{2} \mathrm{O} \nu_{3}$ monomer to lower frequencies. In a mixture $\mathrm{H}_{2} \mathrm{O}: \mathrm{CO}: \mathrm{O}_{2}=1: 50: 50$ the position of the $\nu_{3}$ monomer is shifted by $40 \mathrm{~cm}^{-1}$ to lower frequencies compared to a $\mathrm{H}_{2} \mathrm{O} / \mathrm{O}_{2}$ matrix and $14 \mathrm{~cm}^{-1}$ compared to a $\mathrm{H}_{2} \mathrm{O} / \mathrm{CO}$ matrix. Line broadening increases strongly (see Table 2). In contrast, the bending mode in mixtures containing equal amounts of $\mathrm{CO}$ and $\mathrm{O}_{2}$ is shifted $7 \mathrm{~cm}^{-1}$ towards higher frequencies. Furthermore, the monomeric and dimeric bending modes are blended together in those mixtures. The band width increases strongly in multicomponent mixtures and the band 


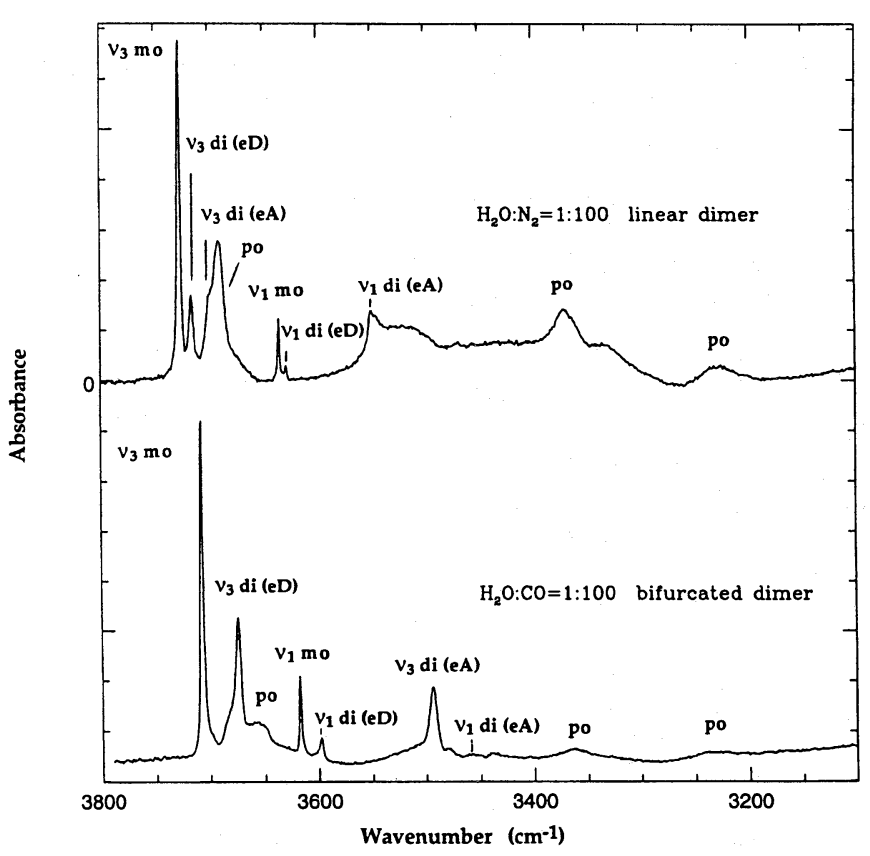

Fig. 2. The infrared spectroscopic pattern of isolated water resulting from a linear (top) or bifurcated (bottom) structure of the $\mathrm{H}_{2} \mathrm{O}$ dimer in the matrix. Symbols $\nu_{3}$ and $\nu_{1}$ refer to the asymmetric and the symmetric $\mathrm{OH}$ stretching mode, respectively. "mo", "di" and "po" denote $\mathrm{H}_{2} \mathrm{O}$ monomers, dimers and polymers. The infrared spectra of dimers display two sets of absorption peaks. "eA" and "eD" are as defined in Fig. 1.

ratio of dimers and multimers to monomers is much higher in mixtures containing mainly $\mathrm{CO}$ and $\mathrm{O}_{2}$ (see Table 2 and 3). This offers a good criterium for the identification of such a particular ice composition, but will strongly depend on the exact $\mathrm{H}_{2} \mathrm{O}$ content. We observe in Fig. 5, Table 1 and 2 that $\mathrm{N}_{2}$ acts as a "silent" participant, when present along with $\mathrm{CO}$ and $\mathrm{O}_{2}$, adding to line broadening but not to changes in band position or infrared pattern.

Solid oxygen is a special molecular crystal (Jansen, 1987). It possesses a triplet electronic spin momentum in its ground state and can therefore behave as a magnetic material. $\mathrm{N}_{2}$ and $\mathrm{CO}$ have identical crystal structure at low temperatures and nearly equal site dimensions (Hagen \& Tielens 1981). Therefore CO and $\mathrm{N}_{2}$ co-crystallize easily and preferentially on a local scale, whereas the particular shape of $\mathrm{O}_{2}$ may prevent such an arrangement. The strong red-shift of all bands in the stretching mode region indicates that a matrix environment of $\mathrm{CO} / \mathrm{O}_{2}$ is much more polarizable than a $\mathrm{CO}$ or $\mathrm{O}_{2}$ matrix alone, which will help to identify indirectly molecular oxygen on grain mantles.

\subsection{Infrared spectroscopy upon diffusion}

During a temperature rise volatile components evaporate from the ice matrix and the matrix properties are changed. These changes can be monitored by infrared spectroscopy. We studied the special behaviour of $\mathrm{H}_{2} \mathrm{O}$ in a $\mathrm{CO} / \mathrm{O}_{2}$ matrix during warmup. Fig. 6 shows the change in the spectrum during a warm-up

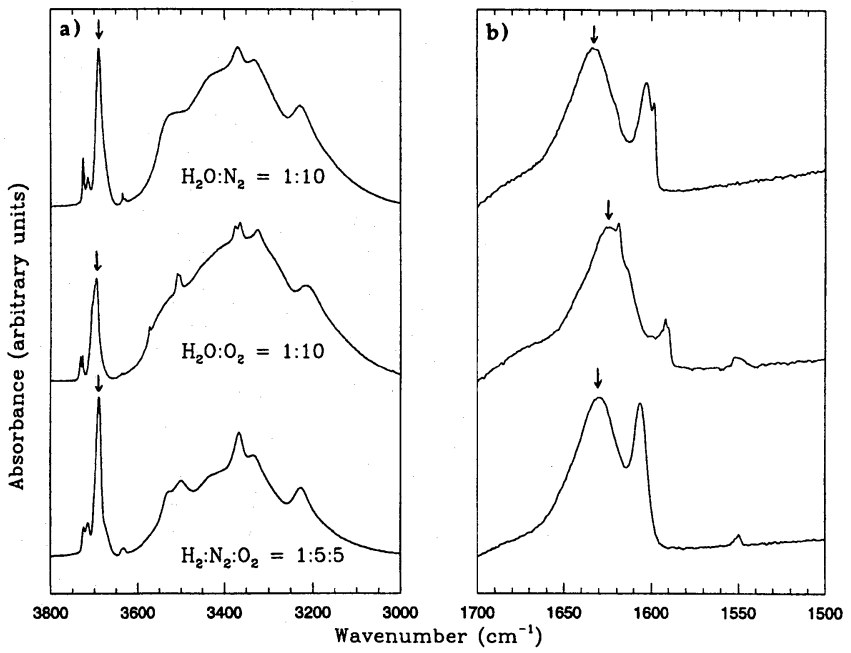

Fig. 3a and b. Infrared absoption spectra of isolated $\mathrm{H}_{2} \mathrm{O}$ in the a stretching 3800-3000 $\mathrm{cm}^{-1}$ and $\mathbf{b}$ bending mode region $1700-1500$ $\mathrm{cm}^{-1}$ in solid $\mathrm{N}_{2}$ and $\mathrm{O}_{2}$ with an absorber to matrix ratio of $1: 10$. In this concentration the dominant bands are due to water multimers, marked with arrows. Band assignments of monomers and dimers are listed in Table 1. a The spectra show a broad underlying component of polymeric water from $3600-3200 \mathrm{~cm}^{-1}$. Bands at high frequencies around $3700 \mathrm{~cm}^{-1}$ are due to water monomers, dimers and small multimers. The bands superimposed on the broad polymeric water feature are due to higher multimers. The dominant sharp peak in all spectra marked with an arrow - is due to small $\mathrm{H}_{2} \mathrm{O}$ multimers (e.g. triplets and tetramers) and lies at $3689 \mathrm{~cm}^{-1}$ in a $\mathrm{H}_{2} \mathrm{O} / \mathrm{N}_{2}$ and $\mathrm{H}_{2} \mathrm{O} / \mathrm{N}_{2} / \mathrm{O}_{2}$ mixture and at $3695 \mathrm{~cm}^{-1}$ in a $\mathrm{H}_{2} \mathrm{O} / \mathrm{O}_{2}$ mixture. $b$ In the bending mode region the broad band around $1630 \mathrm{~cm}^{-1}$ is due to polymeric water. The remaining bands are produced by $\mathrm{H}_{2} \mathrm{O}$ monomers, dimers and small multimers. In a $\mathrm{H}_{2} \mathrm{O} / \mathrm{N}_{2} / \mathrm{O}_{2}$ mixture all these bands are blended in one strong band, centered at $1606 \mathrm{~cm}^{-1}$. The weak fundamental transition of $\mathrm{O}_{2}$ is observed at $1550 \mathrm{~cm}^{-1}$.

sequence. In this particular mixture the monomeric and dimeric water bands already disappear during a temperature rise to 28 $\mathrm{K}$, although the $\mathrm{CO} / \mathrm{O}_{2}$ matrix has not evaporated yet at this temperature. The strong band at $3695 \mathrm{~cm}^{-1}$ is due to small water multimers. At $45 \mathrm{~K}$ the matrix components $\mathrm{CO}$ and $\mathrm{O}_{2}$ have vaporized and water is completely polymerized, showing a broad feature centered at $3350 \mathrm{~cm}^{-1}$. Comparing these spectra to other ice mixtures in Fig. 7 shows that the abundance of multimers is increasing and those of monomers is slightly reduced at $28 \mathrm{~K}$ in a $\mathrm{H}_{2} \mathrm{O} / \mathrm{CO}$ and $\mathrm{H}_{2} \mathrm{O} / \mathrm{O}_{2}$ matrix or when $\mathrm{O}_{2}$ is a minor constituent in a $\mathrm{H}_{2} \mathrm{O} / \mathrm{CO} / \mathrm{O}_{2}$ matrix (compare Fig. 3, 4 and 5 displaying spectra at $10 \mathrm{~K}$ ). However, when the matrix contains $\mathrm{CO}$ and $\mathrm{O}_{2}$ in equal amounts or more $\mathrm{O}_{2}$ than $\mathrm{CO}$ no monomeric or dimeric bands are observed anymore at a temperature of $28 \mathrm{~K}$. This indicates that the crystallization in these ice mixtures are more random on a local scale and that the matrix is easier destroyed. A possibly lower density structure of this ice mixture allows $\mathrm{H}_{2} \mathrm{O}$ to diffuse more freely during a small rise of temperature, resulting in a spectrum which shows even at low temperatures only multimeric bands. Therefore only low temperature ice mantles, which have not experienced any heat- 


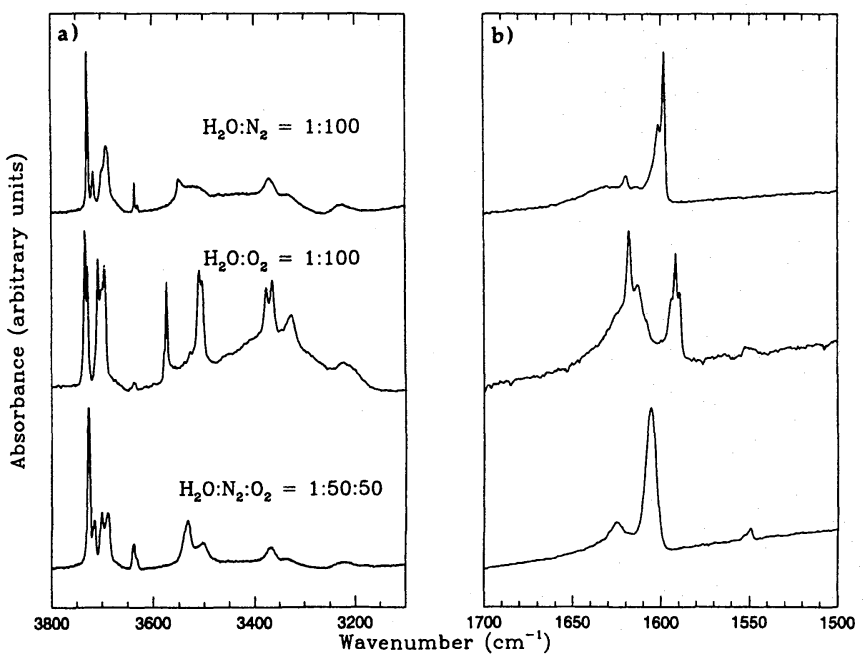

Fig. 4a and b. Infrared absoption spectra of isolated $\mathrm{H}_{2} \mathrm{O}$ in the a stretching $3800-3000 \mathrm{~cm}^{-1}$ and $\mathbf{b}$ bending mode region $1700-1500$ $\mathrm{cm}^{-1}$ in solid $\mathrm{N}_{2}$ and $\mathrm{O}_{2}$ with an absorber to matrix ratio of $1: 100$. In this dilution the dominant bands are due to water monomers. Band assignments of monomers and dimers are listed in Table 1. Note that the band positions of isolated water features remain constant in mixtures of different concentration for identical composition (compare Fig. 3). The spectrum of isolated $\mathrm{H}_{2} \mathrm{O}$ in solid $\mathrm{O}_{2}$ is characterized by a different intensity ratio of $\nu_{3}$ monomeric and dimeric absorption, the absence of $\nu_{1}$ monomer and dimers, and the presence of strong triplets at 3700/3594 $\mathrm{cm}^{-1}\left(\nu_{3}\right)$ and $3509 / 3502 \mathrm{~cm}^{-1}\left(\nu_{1}\right)$.

ing events will display the signature of isolated water ice bands in dense clouds.

\subsection{Infrared spectroscopy upon UV photolysis}

We have also studied the changes of isolated water in various matrices upon irradiation with UV photons. Fig. 8 shows a mixture of $\mathrm{H}_{2} \mathrm{O}: \mathrm{CO}: \mathrm{O}_{2}=1: 50: 50$ irradiated with a microwave discharge lamp over a timescale of 1 hour. Laboratory studies have shown that the molecule $\mathrm{CO}_{2}$ is formed readily upon UV irradiation (d'Hendecourt et al. 1986). The $\mathrm{CO}_{2}$ molecule has two combination modes at $3708 \mathrm{~cm}^{-1}\left(\nu_{1}+\nu_{3}\right.$ combination mode) and at $3600 \mathrm{~cm}^{-1}\left(2 \nu_{2}+\nu_{3}\right.$ combination mode $)$ with infrared band intensities of $1.4 \times 10^{-18}$ and $4.5 \times 10^{-19} \mathrm{~cm} \mathrm{molec}^{-1}$, respectively (Gerakines et al. 1995). These modes increase rapidly upon irradiation. Fig. 8 shows that after 1 min UV irradiation the monomeric features are decreasing and multimers are created. The $\mathrm{CO}_{2}$ combination modes are dominant after 1 min irradiation and after $10 \mathrm{~min}$ UV photolysis no discrete sharp bands due to isolated water ice are present anymore. After one hour of UV irradiation all isolated water is converted to polymeric water ice, which appears as a broad band centered at $3300 \mathrm{~cm}^{-1}$. We have studied several mixtures upon UV irradiation and observe a decrease in band intensity of the monomeric feature after $1 \mathrm{~min}$ $\mathrm{UV}$ irradiation in $\mathrm{H}_{2} \mathrm{O} / \mathrm{CO}, \mathrm{H}_{2} \mathrm{O} / \mathrm{O}_{2}$ and $\mathrm{H}_{2} \mathrm{O} / \mathrm{CO} / \mathrm{O}_{2}$ mixtures but not in a matrix containing $\mathrm{H}_{2} \mathrm{O} / \mathrm{N}_{2}$. This effect is illustrated in Fig. 9 which compares spectra of deposited and irradiated samples. Whereas the spectrum of a mixture of $\mathrm{H}_{2} \mathrm{O} / \mathrm{N}_{2}$ shows
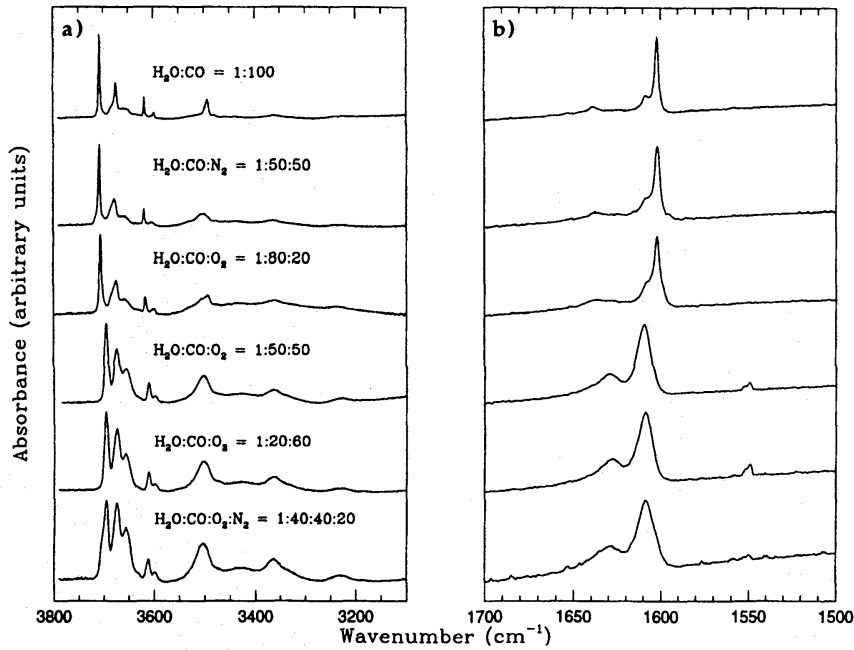

Fig. 5a and b. Infrared absorption spectra of isolated $\mathrm{H}_{2} \mathrm{O}$ in the a stretching $3800-3000 \mathrm{~cm}^{-1}$ and $\mathbf{b}$ bending mode region $1700-1500$ $\mathrm{cm}^{-1}$ in various mixtures containing $\mathrm{CO}, \mathrm{N}_{2}$ and $\mathrm{O}_{2}$. Band assignments of monomers and dimers are listed in Table 1. A strong shift of the $\nu_{3}$ monomer towards lower frequencies (higher frequencies) in the stretching mode (bending mode) regions is observed in mixtures containing equal amounts of $\mathrm{CO}$ and $\mathrm{O}_{2}$. Furthermore, monomeric and dimeric absorptions are blended in one strong band in such mixtures in the bending mode region. The weak fundamental transition of $\mathrm{O}_{2}$ is observed at $1550 \mathrm{~cm}^{-1}$.

hardly any change after 1 min UV irradiation, the spectrum of a $\mathrm{H}_{2} \mathrm{O} / \mathrm{CO}$ mixture shows a decrease in all monomeric and dimeric bands of about $40 \%$. The decrease in band strength of the $\nu_{3}$ monomer in mixtures containing $\mathrm{H}_{2} \mathrm{O} / \mathrm{O}_{2}, \mathrm{H}_{2} \mathrm{O} / \mathrm{CO} / \mathrm{O}_{2}$ and $\mathrm{H}_{2} \mathrm{O} / \mathrm{N}_{2} / \mathrm{O}_{2}$ is $55 \%, 40 \%$ and $40 \%$, respectively. UV irradiation changes the matrix properties and destroys sites for isolated water. The presence of $\mathrm{CO}$ and/or $\mathrm{O}_{2}$ rapidly creates new irradiation products, whereas $\mathrm{N}_{2}$ is rather unreactive. Sites for isolated water ice are therefore longer preserved in a $\mathrm{N}_{2}$ matrix as shown in Fig. 9. These results indicate that the search for water ice in isolated form will be rather difficult on photoprocessed grains.

\subsection{Infrared band strength of isolated $\mathrm{H}_{2} \mathrm{O}$}

We have also performed specific measurements in order to determine the integrated absorbance of monomeric and dimeric water ice in $\mathrm{CO}, \mathrm{O}_{2}$ and $\mathrm{N}_{2}$ matrices, following the method of Gerakines et al. (1995). For this experiment we deposited $\mathrm{H}_{2} \mathrm{O}$ and the matrix gas (e.g. $\mathrm{CO}$ ) through two different deposition tubes. In the first experiment water was deposited together with a large amount of $\mathrm{CO}$. In the second experiment only water was deposited, yielding a polymeric signature with well known intensity (e.g. d'Hendecourt \& Allamandola 1986). Because both experiments were performed under the same conditions, the water abundance in both experiments has to be equal, allowing us to obtain the combined column density of monomers, dimers and multimers. We used a lattice model with coordination number 12 for the probability of occurrence of a $\mathrm{H}_{2} \mathrm{O}$ molecule in 


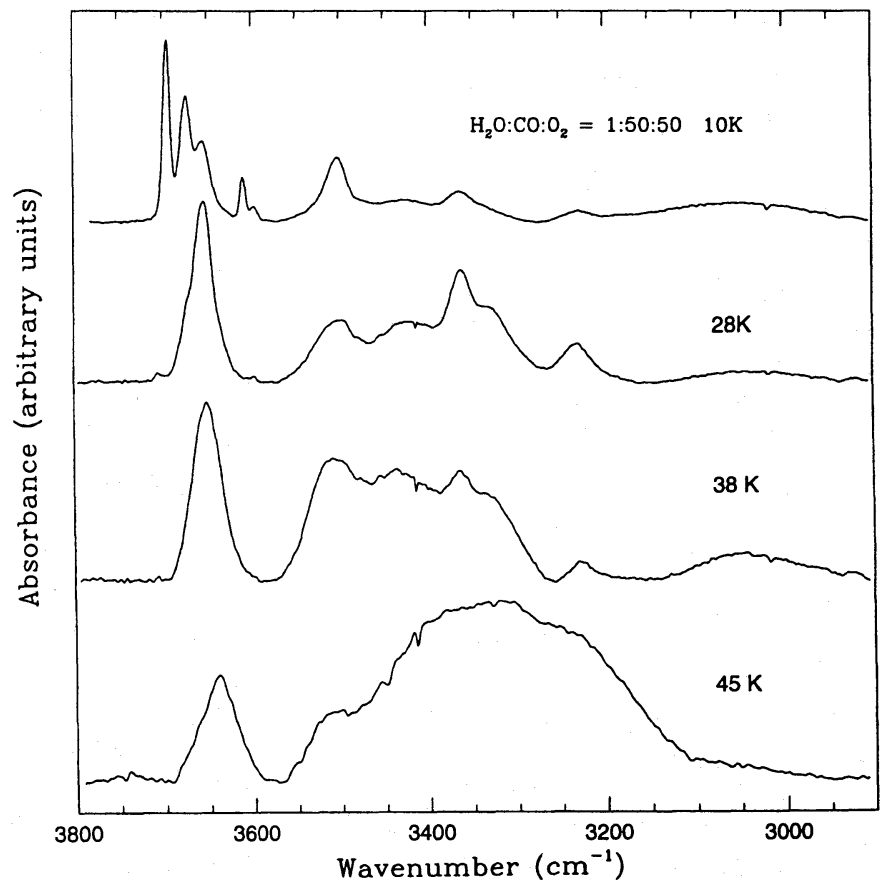

Fig. 6. Infrared absorption spectra of isolated $\mathrm{H}_{2} \mathrm{O}$ in the stretching $3800-3000 \mathrm{~cm}^{-1}$ mode region in a mixture containing $\mathrm{H}_{2} \mathrm{O} / \mathrm{CO} / \mathrm{O}_{2}=1$ :50:50 during warm up. The evaporation of volatile matrix constituents during temperature rise results in destruction of the ice matrix and the disappearance of monomers and dimers. At $45 \mathrm{~K}$ only multimeric water remains at $3695 \mathrm{~cm}^{-1}$ (small $\mathrm{H}_{2} \mathrm{O}$ multimers) and $3350 \mathrm{~cm}^{-1}$ (polymeric $\mathrm{H}_{2} \mathrm{O}$ ).

monomeric and dimeric form (Flory 1942, Huggins 1942). According to these models we assume that $85 \%$ of water will be in monomeric form when the absorber to matrix ratio is $1: 100$, about $12 \%$ of water will be in dimeric form and the rest in multimers. The values for the integrated absorbance of monomers and dimers in $\mathrm{CO}, \mathrm{N}_{2}$ and $\mathrm{O}_{2}$ matrices are summarized in Table 5. In a $\mathrm{H}_{2} \mathrm{O} / \mathrm{CO}$ mixture the integrated absorbance derived for the $\nu_{3}$ monomer is $1.1 \times 10^{-17} \mathrm{~cm} \mathrm{molec}^{-1}$, a factor 18 reduced compared to polymeric water $\left(2.0 \times 10^{-16} \mathrm{~cm} \mathrm{molec}^{-1}\right)$. As a typical case the column density of solid CO in NGC 7538 IRS 9 in a non-polar environment has been derived by Tielens et al. (1991) to be $6.4 \times 10^{17} \mathrm{~cm}^{-2}$. For the field star Elias 16 a nonpolar CO column density of $4 \times 10^{17} \mathrm{~cm}^{-2}$ has been estimated. Table 5 indicates that water ice can be detected when present as a minor species on a $1 \%$ level relative to $\mathrm{CO}$ in non-polar ices (yielding an equivalent width of a few $\%$, corresponding to an optical depth $\tau \approx 0.01$ ).

\subsection{Isolated water and solid $\mathrm{CO}_{2}$}

The molecule $\mathrm{CO}_{2}$ is efficiently produced by UV photolysis of grain mantles containing $\mathrm{CO}$ (d'Hendecourt et al. 1986). $\mathrm{CO}_{2}$ is not observable from the ground. The detection of solid $\mathrm{CO}_{2}$ through its weak $\nu_{2}$ bending mode at $15.2 \mu \mathrm{m}$ was reported by d'Hendecourt \& de Muizon (1989) from IRAS-LRS spectra in 3 sources. The abundance of solid $\mathrm{CO}_{2}$ toward AFGL 961 was

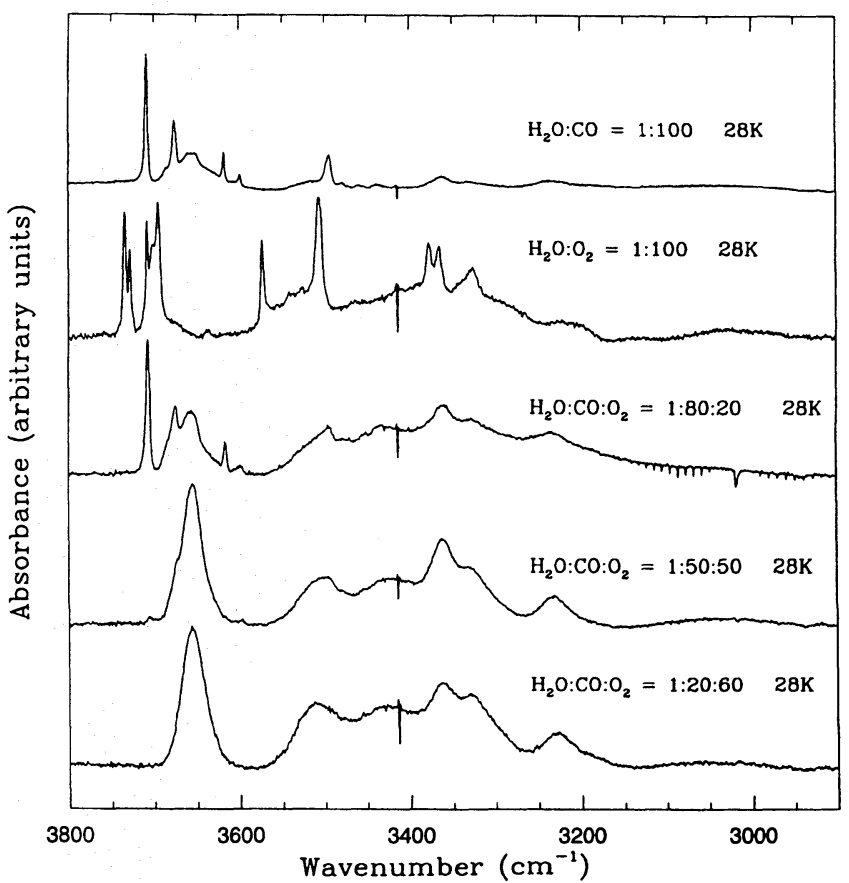

Fig. 7. Infrared absorption spectra of isolated $\mathrm{H}_{2} \mathrm{O}$ in the stretching mode region $\left(3800-3000 \mathrm{~cm}^{-1}\right)$ in various mixtures warmed up to 28 $\mathrm{K}$. A matrix containing equal amounts of $\mathrm{CO}$ and $\mathrm{O}_{2}$ is faster destroyed upon rise of temperature. Subsequently $\mathrm{H}_{2} \mathrm{O}$ monomers and dimers are not observed.

estimated to be $10 \%$ of $\mathrm{H}_{2} \mathrm{O}$. A search of this band towards a number of other embedded sources yielded $\mathrm{CO}_{2}$ upper limits of 1-3\% relative to $\mathrm{H}_{2} \mathrm{O}$ (Whittet \& Walker 1991). Reanalysis of LRS spectra indicate that $\mathrm{CO}_{2}$ is a widespread component in interstellar ices (d'Hendecourt \& Ehrenfreund, 1996).

The combination modes of $\mathrm{CO}_{2}$ at 3708 and $3600 \mathrm{~cm}^{-1}$ will interfere with the $\nu_{3}$ and $\nu_{1}$ modes of isolated $\mathrm{H}_{2} \mathrm{O}$. For this reason it is important to investigate the constraints which solid $\mathrm{CO}_{2}$ poses on the detection of isolated $\mathrm{H}_{2} \mathrm{O}$ features. We have therefore performed experiments including $\mathrm{CO}_{2}$ along with $\mathrm{CO}$, $\mathrm{N}_{2}$ and $\mathrm{O}_{2}$. Fig. 10a shows a mixture of $\mathrm{H}_{2} \mathrm{O} / \mathrm{CO}_{2}=1: 100$ displaying the two strong combination modes of $\mathrm{CO}_{2}$ at 3708 and $3600 \mathrm{~cm}^{-1}$. Several weak bands can be seen between the two strong $\mathrm{CO}_{2}$ bands, which cannot be identified unambiguously, because other bands may be obscured by the strong $\mathrm{CO}_{2} \mathrm{com}$ bination modes. Experiments using $\mathrm{D}_{2} \mathrm{O}$ are needed to identify the monomers and dimers of $\mathrm{H}_{2} \mathrm{O}$. In a mixture of non-polar ices including $10 \% \mathrm{CO}_{2}$, shown in Fig. 10b, no discrete bands of isolated water are observed and only one broad component, likely a blend of various features, appears as a shoulder on the low frequency side of the strong $\mathrm{CO}_{2}$ band at $3708 \mathrm{~cm}^{-1}$. In a matrix containing $\mathrm{CO}, \mathrm{O}_{2}, \mathrm{~N}_{2}$ with $\mathrm{CO}_{2}$ as a minor species of $1 \%$ (see Fig. 10c), the monomers and dimers could be well identified and are listed in Table 1. We conclude that the presence of amounts of $\mathrm{CO}_{2}$ in excess of $5 \%$ on grain mantles renders the search for isolated $\mathrm{H}_{2} \mathrm{O} \nu_{3}$ and $\nu_{1}$ features rather difficult. It must be noted however, that $\mathrm{CO}_{2}$ will have no influence on the detectability of the $\nu_{2}$ bending mode. 


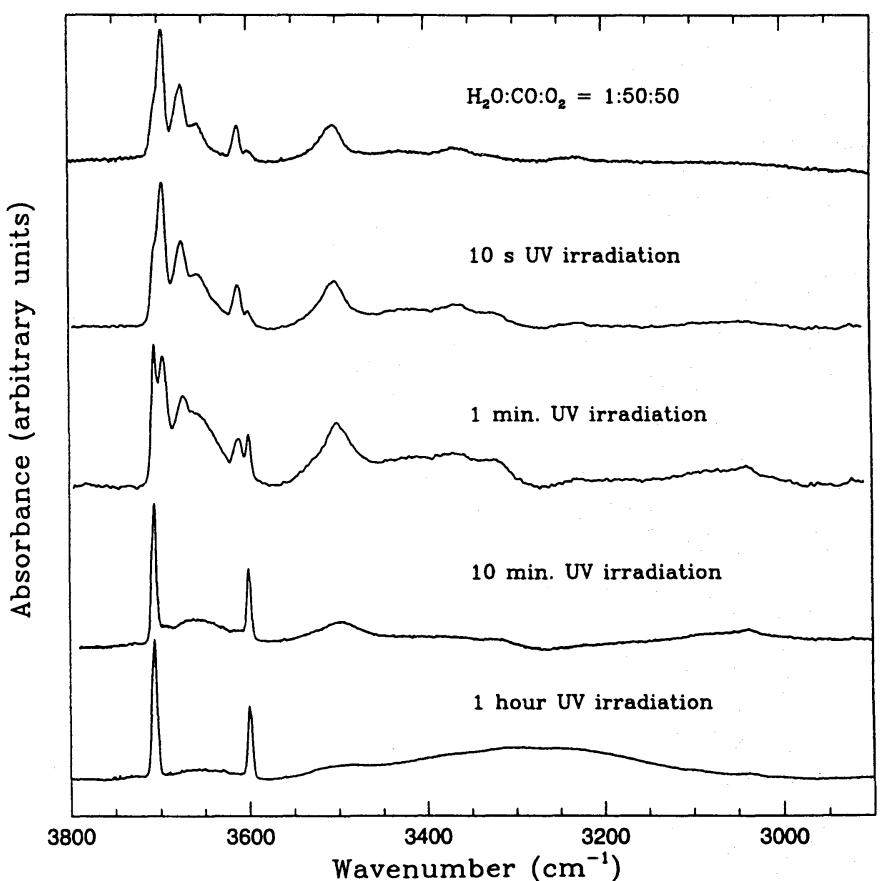

Fig. 8. Infrared absorption spectra of isolated $\mathrm{H}_{2} \mathrm{O}$ in the stretching mode region ( $3800-3000 \mathrm{~cm}^{-1}$ ) in a $\mathrm{H}_{2} \mathrm{O} / \mathrm{CO} / \mathrm{O}_{2}=1: 50: 50$ ice mixture. UV irradiation was performed for a duration of one hour. The matrix is destroyed upon UV photolysis and isolated water is rapidly converted into polymeric water. The spectra are dominated by the strong combination modes of the photoproduct $\mathrm{CO}_{2}$ at 3708 and $3600 \mathrm{~cm}^{-1}$.

\subsection{Isolated water diluted in polycyclic aromatic hydrocarbons}

Finally, we have studied isolated water in a matrix containing naphthalene. PAHs are thought to be an abundant component of the interstellar medium (Puget \& Leger, 1989). It is certainly not a realistic case that water will be isolated in naphthalene on interstellar grains. However, for practical reasons we have chosen naphthalene as a model polycyclic aromatic hydrocarbon (PAH), since larger PAHs are not volatile at room temperature. A matrix containing PAHs will exhibit no crystalline ordered structure but a random orientation. Naphthalene crystals will show an open and low density structure and act as a strong perturbing medium. The abundance of naphthalene in our experiments was calculated using the integrated intensity by Szczepanski et al. (1992). The bands due to isolated water are shown in Fig. 11a and are tentatively identified as the $\nu_{3}$, $\nu_{1}$ and $\nu_{2}$ monomers at 3654,3571 and $1630 \mathrm{~cm}^{-1}$, respectively. This identification seems plausible, since the band ratio remains constant in experiments with different $\mathrm{C}_{10} \mathrm{H}_{8}$ concentrations. The band width are $48 \mathrm{~cm}^{-1}\left(\lambda 3654 \mathrm{~cm}^{-1}\right), 44 \mathrm{~cm}^{-1}$ $\left(\lambda 3571 \mathrm{~cm}^{-1}\right)$ and $7 \mathrm{~cm}^{-1}\left(\lambda 1630 \mathrm{~cm}^{-1}\right)$. Due to the large size of the naphthalene molecule the water molecules are completely isolated. The bands show a strong red-shift (blue-shift) in the stretching (bending) mode region compared to gas phase $\mathrm{H}_{2} \mathrm{O}$, indicative of a strong perturbation of the matrix. The polarizibility of aromatic species is generally large, e.g. for benzene a value of $\alpha=25.1 \times 10^{-24} \mathrm{~cm}^{-3}$ is measured. The numerous bands

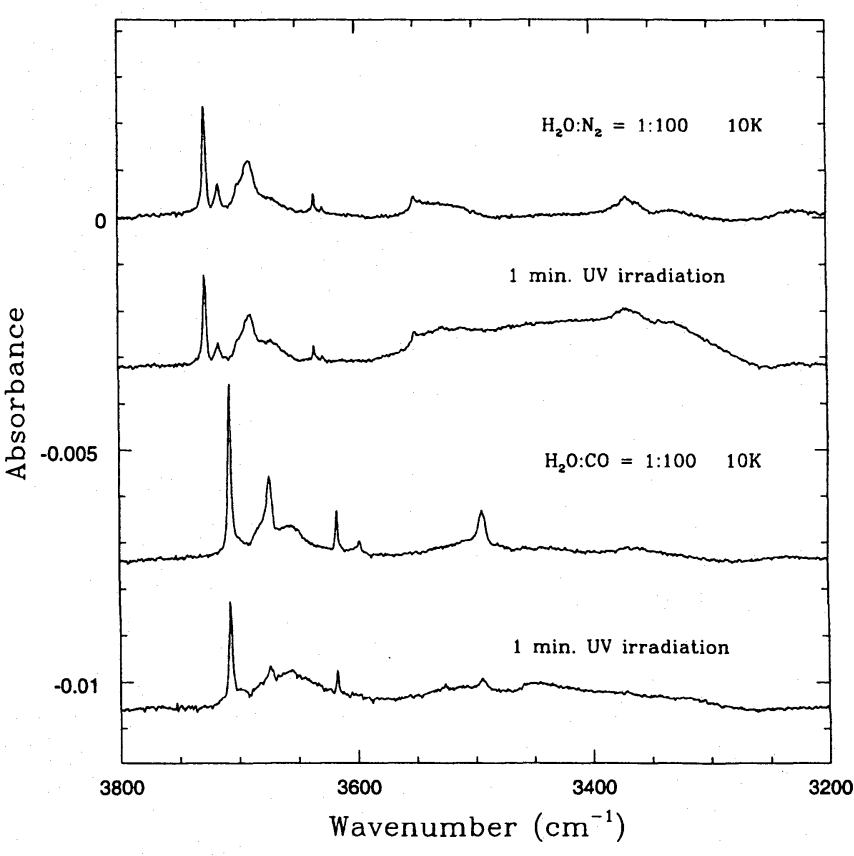

Fig. 9. Infrared absorption spectra of isolated $\mathrm{H}_{2} \mathrm{O}$ in the stretching mode region (3800-3000 cm-1) diluted in a $\mathrm{H}_{2} \mathrm{O} / \mathrm{N}_{2}$ and $\mathrm{H}_{2} \mathrm{O} / \mathrm{CO}$ mixture after $1 \mathrm{~min}$ UV photolysis. Whereas no change in the spectrum of a $\mathrm{H}_{2} \mathrm{O} / \mathrm{N}_{2}$ matrix is observed, a decrease of $40 \%$ in the $\nu_{3}$ monomer and $49 \%$ in the $\nu_{3}$ dimer is observed in the $\mathrm{H}_{2} \mathrm{O} / \mathrm{CO}$ ice mixture after short irradiation.

around 3050 and $1500 \mathrm{~cm}^{-1}$ are exclusively due to $\mathrm{C}-\mathrm{H}$ and C-C stretching modes of naphthalene (Pauzat et al. 1992). We have further performed an experiment where $\mathrm{CO}$ is the main component and a small amount of naphthalene and water are mixed in the same matrix, shown in Fig. 11b. This mixture is more closely related to interstellar conditions. The same pattern of bands as seen in Fig. 11a is observed shifted to higher frequencies (indicating the presence of $\mathrm{CO}$ ) with small shoulders appearing on the main bands of isolated water ice, which are probably due to the monomers surrounded by naphthalene molecules. We identify the bands in Fig. 11b as the $\nu_{3}$ and $\nu_{1}$ monomer in $\mathrm{CO}$ at 3689 and $3603 \mathrm{~cm}^{-1}$, and as the $\nu_{3}$ and $\nu_{1}$ monomer in naphthalene at 3670 and $3595 \mathrm{~cm}^{-1}$, respectively. The $\nu_{2}$ monomer appears as one broad feature at $1609 \mathrm{~cm}^{-1}$. These experiments should be taken as a fundamental study of matrix effects and the derived values of band positions should give an idea of the possible spectroscopic pattern (including shifts and broadening) which can be expected for grain mantles which contain such polarizable molecules.

\section{Discussion}

Isolated water was already previously investigated in the context of hydrogen bonding in individual matrices such as $\mathrm{Ar}, \mathrm{CO}$ and $\mathrm{N}_{2}$ (Benedict et al. 1956, Mann et al. 1974, Barnes et al. 1980, Fredin et al. 1977, Murby et al. 1979, Hagen \& Tielens 1982, Tso \& Lee 1985). We present for the first time a comprehensive study of water diluted in various ice mixtures, likely represen- 


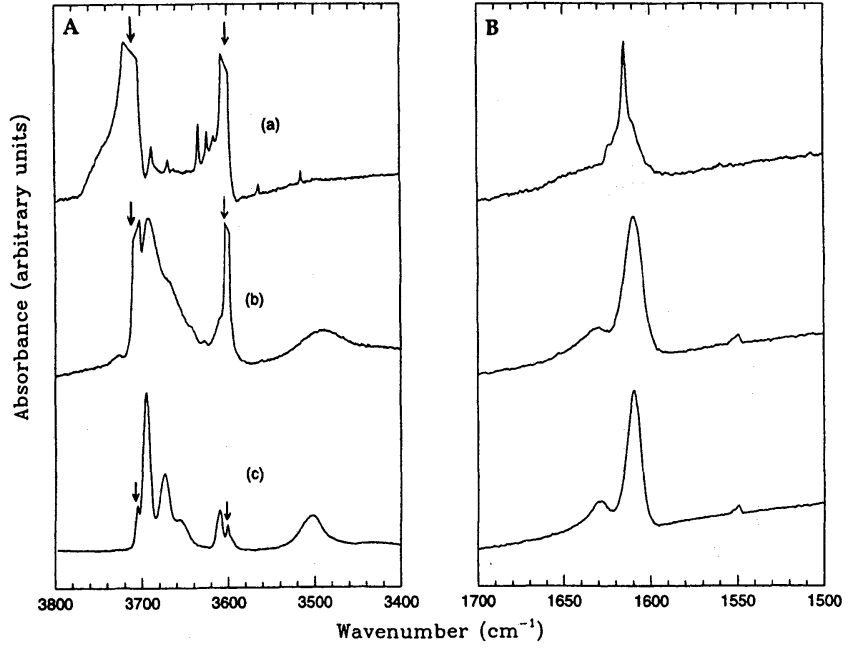

Fig. 10A and B. Infrared absorption spectra of isolated $\mathrm{H}_{2} \mathrm{O}$ in the A stretching (3800-3400 $\mathrm{cm}^{-1}$ ) and $\mathbf{B}$ bending mode region (1700-1500 cm $\mathrm{cm}^{-1}$ ) in a) a matrix $\mathrm{H}_{2} \mathrm{O} / \mathrm{CO}_{2}=1: 100 \mathrm{~b}$ ) a matrix containing $\mathrm{H}_{2} \mathrm{O}: \mathrm{CO}: \mathrm{O}_{2}: \mathrm{CO}_{2}: \mathrm{N}_{2}=1: 25: 25: 10: 10$. The strong combination modes of $\mathrm{CO}_{2}$ are truncated and marked with an arrow. No identification of isolated water bands is possible in this mixture due to the strong $\mathrm{CO}_{2}$ combination modes at 3708 and $3600 \mathrm{~cm}^{-1}$.

c) a matrix containing $\mathrm{H}_{2} \mathrm{O}: \mathrm{CO}: \mathrm{O}_{2}: \mathrm{CO}_{2}: \mathrm{N}_{2}=1: 50: 35: 1: 15$. Small concentrations of $\mathrm{CO}_{2}$ do permit the identification of isolated water ice (see Table 1).

Table 5. Infrared band intensities of isolated $\mathrm{H}_{2} \mathrm{O}$ in various matrices.

\begin{tabular}{lll}
\hline Ice matrix & Band & $\begin{array}{l}\mathrm{A} \\
\mathrm{cm} \mathrm{molec}\end{array}$ \\
\hline $\mathrm{H}_{2} \mathrm{O}: \mathrm{CO}=1: 100$ & $\nu_{3}$ monomer & $1.1 \times 10^{-17}$ \\
& $\nu_{3}$ dimer "eD" & $5.8 \times 10^{-17}$ \\
& $\nu_{2}$ monomer & $3.5 \times 10^{-18}$ \\
& $\nu_{2}$ dimer "eD" & $1.0 \times 10^{-17}$ \\
$\mathrm{H}_{2} \mathrm{O}: \mathrm{O}_{2}=1: 100$ & $\nu_{3}$ monomer & $3.3 \times 10^{-18}$ \\
& $\nu_{3}$ dimer "eD" & $1.5 \times 10^{-17}$ \\
& $\nu_{2}$ monomer & $8.4 \times 10^{-19}$ \\
& $\nu_{2}$ dimer "eD" & $9.0 \times 10^{-18}$ \\
& $\nu_{2}$ dimer "eA" & $1.7 \times 10^{-17}$ \\
$\mathrm{H}_{2} \mathrm{O}: \mathrm{N}_{2}=1: 100$ & $\nu_{3}$ monomer & $7.8 \times 10^{-18}$ \\
& $\nu_{3}$ dimer "eD" & $1.5 \times 10^{-17}$ \\
& $\nu_{2}$ monomer & $6.1 \times 10^{-18}$ \\
& $\nu_{2}$ dimer "eD" & $2.4 \times 10^{-17}$ \\
\hline
\end{tabular}

tative of interstellar grain mantles. We have measured the main features of isolated water, monomers and dimers, in different ice mixtures and concentrations in order to determine bands that can be detected by astronomical observations. Furthermore we measured the integrated absorbance of $\mathrm{H}_{2} \mathrm{O}$ monomers and dimers in $\mathrm{CO}, \mathrm{N}_{2}$ and $\mathrm{O}_{2}$.

There is strong astronomical evidence for the existence of non-polar ices due to the observed CO profiles ( Sandford et al. 1988, Tielens et al. 1991, Chiar et al. 1994). In polar ices,

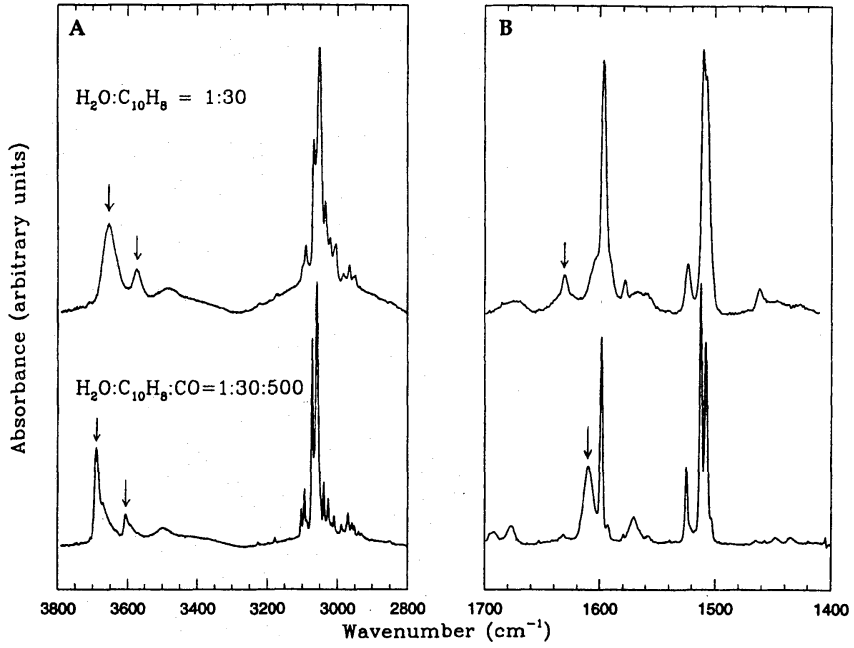

Fig. 11A and B. Infrared absorption spectra of isolated $\mathrm{H}_{2} \mathrm{O}$ in the A stretching (3800-2800 $\mathrm{cm}^{-1}$ ) and $\mathbf{B}$ bending mode region (1700-1400 $\mathrm{cm}^{-1}$ ) in a) a $\mathrm{C}_{10} \mathrm{H}_{8}$ matrix and b) a matrix containing $\mathrm{H}_{2} \mathrm{O} / \mathrm{C}_{10} \mathrm{H}_{8} / \mathrm{CO}$ $=1: 30: 500$. The band pattern centered at 3050 and $1510 \mathrm{~cm}^{-1}$ is due to the $\mathrm{C}-\mathrm{H}$ and $\mathrm{C}-\mathrm{C}$ stretching modes of naphthalene. The spectra show only $\mathrm{H}_{2} \mathrm{O}$ monomers - marked with an arrow, as described in the text and show a strong red-shift in the stretching mode region due to the polarizable medium.

which can survive on grains relatively close to the star, water ice is in polymeric form and the most abundant constituent. Therefore isolated water features can be detected only in nonpolar ices, which can only survive far from the star. We studied the behaviour of isolated water in the particular context of nonpolar ices.

Isolated water bands can be well distinguished in each individual matrix like $\mathrm{CO}, \mathrm{N}_{2}$ or $\mathrm{O}_{2}$. However, "dirty ices", containing a mixture of these molecules, are expected on interstellar grains. The overall spectroscopic pattern of isolated water including stretching and bending vibrations may allow us to infer the non-polar ice constituents. For a typical absorber to matrix ratio of $1: 100,85 \%$ of the water molecules are statistically ordered in a monomeric structure, while $12 \%$ are contained in dimer structures. Even for mixtures with absorber to matrix ratio equal 1:10 the monomer and dimer contributions are 32 $\%$ and $13 \%$, respectively. Monomers and dimers $\left(\nu_{3}, \nu_{2}\right.$ and $\nu_{1}$ ) show sharp bands which are quite sensitive to the matrix environment.

Multimer assignments are speculative and an unambiguous identification is rather difficult. We have therefore concentrated on the interpretation of the $\nu_{3}$ asymmetric stretching and the $\nu_{2}$ bending mode of the $\mathrm{H}_{2} \mathrm{O}$ monomer and dimer to constrain the composition of non-polar mixtures. Our results imply that in case water is a minor constituent in the matrix (absorber to matrix ratio equal 1:100) it will be possible to detect the $\nu_{3}$ and $\nu_{2}$ monomers and dimers of $\mathrm{H}_{2} \mathrm{O}$ when the major compounds in the matrix are $\mathrm{CO}$, along with $\mathrm{N}_{2}$ and $\mathrm{O}_{2}$. The derived integrated absorbance for the $\nu_{3}$ and $\nu_{2}$ monomeric $\mathrm{H}_{2} \mathrm{O}$ features in 
CO should allow a detection with ISO in lines of sight with a considerable non-polar ice column density $\left(\geq 5 \times 10^{17} \mathrm{~cm}^{-2}\right)$.

The behaviour of isolated water in $\mathrm{CO}$ has been extensively studied by Hagen \& Tielens (1981). When introducing additionally molecular nitrogen in a $\mathrm{CO}$ matrix, the pattern and band position of isolated $\mathrm{H}_{2} \mathrm{O}$ does not alter. The anisotropic field caused by the shape of the $\mathrm{CO}$ molecule induces a dominant interaction with isolated $\mathrm{H}_{2} \mathrm{O}$ molecules, whereas the unreactive $\mathrm{N}_{2}$ molecules play apparently a neutral role in the matrix. The presence of $\mathrm{O}_{2}$ in equal amounts as $\mathrm{CO}$ causes a large shift towards lower frequencies for the $\nu_{3}$ stretching mode of the $\mathrm{H}_{2} \mathrm{O}$ monomer, indicative of a perturbing matrix. From the astronomical point of view this provides a promising diagnostic to trace the infrared inactive molecule $\mathrm{O}_{2}$.

The matrix behaviour of $\mathrm{CO}$ in combination with molecular oxygen also shows a particular result during warm-up and photolytic processes. During warm-up processes the matrix is destroyed due to evaporation of volatile constituents. Hbonding between the water molecules becomes possible, resulting in the formation of multimeric complexes and the absence of monomers and dimers. UV irradiation processes convert isolated water over relatively short timescales into polymeric water. Furthermore the appearance of photolysis products can mask the signature of diluted water ice, e.g. $\mathrm{CO}_{2}$ combination modes at 3708 and $3600 \mathrm{~cm}^{-1}$. These modes effectively obscure the isolated $\mathrm{H}_{2} \mathrm{O}$ features when the $\mathrm{CO}_{2}$ content of the non-polar ice rises above $5 \%$, although the visibility of the $\nu_{2}$ modes is not affected. Therefore the detection of isolated water ice requires cold and UV shielded environments. For this reason the best a priori targets for a search for these features are field stars obscured by a large column of dense cloud material, e.g. Elias 16 in the Taurus cloud $\left(\mathrm{N}(\mathrm{CO})=4 \times 10^{17} \mathrm{~cm}^{-2}\right.$ ) or CK2 in the Serpens cloud $\left(\mathrm{N}(\mathrm{CO})=1.1 \times 10^{18} \mathrm{~cm}^{-2}\right)$. Such objects probe very low temperature dust $(\leq 20 \mathrm{~K})$ in dense clouds with an abundant non-polar ice mantle component (Tielens et al. 1991, Chiar et al. 1994, 1995). Moreover very little UV processing appears to have taken place in these environments (Tegler et al. 1995, Chiar et al. 1995). However, a number of embedded sources may also be suitable candidates. These include NGC 7538/IRS9 $(\mathrm{N}(\mathrm{CO})$ $\left.=6.4 \times 10^{17} \mathrm{~cm}^{-2}\right)$ and L1551/IRS2 $\left(\mathrm{N}(\mathrm{CO})=2.5 \times 10^{17} \mathrm{~cm}^{-2}\right.$, Tielens et al. 1991).

The infrared properties of isolated water have also been studied in a matrix containing naphthalene, in order to determine the changes in band position and overall structure. In such a strong polarizable medium large band shifts are observed due to the interaction of PAHs. Thus features of $\mathrm{H}_{2} \mathrm{O}$ diluted in $\mathrm{PAH}$ dominated ices should be easily distinguishable from other isolated water bands.

\section{Conclusion}

In summary, the infrared spectroscopic study of isolated water under astrophysical conditions has shown that the band position and band shape of water ice are very sensitive to the molecular environment. Isolated water might be present in non-polar ices. Multimeric features cannot be unambiguously identified. Di- luted water ice will therefore provide the best diagnostic for the grain mantle composition when it is present as a minor species (up to a few \%). In this case the asymmetric $\nu_{3}$ stretch of monomeric water is the first and strongest band followed by a typical spectroscopic pattern which results from the specific structure of dimers and trimers in the ice matrix. Together with the bending vibration around $6.0 \mu \mathrm{m}$ these bands are the base for the identification of isolated water. Expecting a dirty ice matrix on grains, containing mainly non-polar $\mathrm{CO}$ and possibly $\mathrm{O}_{2}$ and $\mathrm{N}_{2}$, we may be able to detect isolated water ice and derive its correct abundance. In case the abundance of $\mathrm{O}_{2}$ equals the amount of solid $\mathrm{CO}$ on the grain mantle we can infer the presence of molecular oxygen, since the spectroscopic signature in this case is rather unique. Only grains which contain little (a few \%) $\mathrm{CO}_{2}$ will allow us to measure the isolated water stretching modes in the 2.65-2.78 $\mu \mathrm{m}$ region. However, the $\nu_{2}$ bending modes can be observed independent of the $\mathrm{CO}_{2}$ abundance in the grain mantle. Moreover, warm-up and UV processing efficiently destroy sites for isolated water molecules. Also $\mathrm{CO}_{2}$ is formed by UV radiation on grain surfaces. Therefore warm environments with a large UV field, i.e. close to an embedded star are not suitable for the detection of isolated water. For this reason highly obscured field stars appear to be the best candidates. It is interesting to note that in contrast to the method described here, a direct detection of $\mathrm{O}_{2}$ through its weak absorption at $1550 \mathrm{~cm}^{-1}(6.4$ $\mu \mathrm{m})$ is strongly favored in $\mathrm{CO}_{2}$ rich ices, for which this band is most intense (Ehrenfreund et al. 1992). Recent results show that it will be rather difficult to detect molecular oxygen in the solid phase and thus complementary methods like the band position of isolated water ice and profiles of the $\mathrm{CO}$ and $\mathrm{CO}_{2}$ bands are necessary to trace the infrared molecules $\mathrm{O}_{2}$ and $\mathrm{N}_{2}$ (Ehrenfreund et al. 1996).

The stretching and bending vibrations of isolated $\mathrm{H}_{2} \mathrm{O}$ lie between 2.65 and $2.78 \mu \mathrm{m}$ and 6.15 and $6.3 \mu \mathrm{m}$, respectively. These regions are not observable from the ground. This project is dedicated to the solid state spectroscopy program of Leiden Observatory for the ISO satellite, successfully launched in November 1995, which will measure these wavelength ranges for the first time with sufficiently high sensitivity and resolution. Due to the reduced sensitivity around $2.7 \mu \mathrm{m}$ of the SWS (Short Wavelength Spectrometer) on-board ISO the feasibility of the observations is not guaranteed for very extincted objects, because we expect in the near-infrared a rather weak optical depth for the isolated water bands. However, also valuable information can be retrieved from the observation of isolated water bands in the bending mode region around $6 \mu \mathrm{m}$. The presented results show that we might be able to identify isolated water ice and to infer the non-polar grain mantle composition along the observed lines of sight. The detection of isolated water can provide important insights into interstellar chemistry and will help to confirm the presence of non-polar ices and constrain their $\mathrm{O}_{2}$ content. Such information is essential to understand the chemical and physical conditions which gave rise to the non-polar component in dense cloud ices. The present results highlight that laboratory measurements using solid state spec- 
troscopy are crucial for the identification of the grain mantle composition in dense clouds.

Acknowledgements. We thank Louis d'Hendecourt and Marco Spaans for fruitful discussion and Menno de Groot for technical assistance in the laboratory. PE is a recipient of a fellowship of the European Community ERBCHBICT940939. WS is grateful to ASTRON and SRON for financial support. This work is funded by NASA grants NGR 33018-148 and NAGW 4030.

\section{References}

Barnes A. J., Szczepaniak K., Orville-Thomas W. J., 1980, J.Mol.Struc. 59,39

Benedict W. S., Gailar N., Plyler E. K., 1956, J.Chem.Phys. 24, 1139

Chiar J. E., Adamson A. J., Kerr T. H., Whittet D. C. B., 1994, ApJ 426,240

Chiar J. E., Adamson A. J., Kerr T. H., Whittet D. C. B., 1996, ApJ, in press

d'Hendecourt L. B., Allamandola L. J., Greenberg J. M., 1985, A\&A 152,130

d'Hendecourt L. B., Allamandola L. J., Grim R., Greenberg J. M., 1986, A\&A 158, 119

d'Hendecourt L. B., Jourdain de Muizon M., 1989, A\&A 223, L5

d'Hendecourt L.B., Allamandola L.J., 1986, A\&AS 64, 453

d'Hendecourt L.B., Ehrenfreund P., 1996, Proceedings: "The Role of Dust in the Formation of Stars", ESO, Munich, September 1995, in press

Ehrenfreund P., Breukers R., d'Hendecourt L., Greenberg J. M., 1992, A\&A 260, 431

Ehrenfreund P., Schutte W.A., Gerakines P., van Dishoeck E.F. (1996) in preparation for A\&A

Flory P. J., 1942, J.Chem.Phys 10,51

Fredin L., Nelander B., Ribbegard G., 1977, J.Chem.Phys. 66, 4065

Gerakines P. A., Schutte W. A., Greenberg J. M., van Dishoeck E. F., 1995, A\&A, 296, 810

Greenberg J.M., 1976, Ap\&SS 39, 9

Greenberg J. M., 1982, Comets, ed. Wilkening, L.L. Tucson, University of Arizona Press, 131

Grim R. J. A., d'Hendecourt L., 1986, A\&A 167, 161

Grim R.J.A., Baas F., Geballe T.R., Greenberg J.M., Schutte W., 1991, A\&A 243, 473

Hagen W., Tielens A. G. G. M., 1981, J. Chem. Phys. 75, 4198

Hagen W., Tielens A. G. G. M., Greenberg J. M., 1981, Chem. Phys. 56,367

Hasegawa T.I., Herbst E., 1993, MNRAS 261, 83

Huggins M. L. 1942, J.Phys.Chem 46, 151

Jansen A. P. J. 1987, thesis: Theoretical Approach to the optical, thermodynamic and magnetic properties of solid nitrogen and solid oxygen, University of Nijmegen

Kerr T. H., Adamson A. J., Whittet D. C. B., 1993, MNRAS 262, 1047

Léger A., Jura M., Omont A., 1985, A\&A 144, 147

Mann B., Neikes T., Schmidt E., Luck W., 1974, Phys.Chem. 78, 1236

Morokuma K., Pedersen L., 1968, J.Phys.Chem. 48,7, 3275

Murby E. J., Pullin A. D., 1979, Aust.J.Chem. 32, 1167

Pauzat F., Talbi D., Miller M. D., DeFrees D. J., Ellinger Y., 1992, J.Phys.Chem.96, 7882

Pendleton Y. J., Sandford S. A., Allamandola L. J., Tielens A. G. G. M., Sellgren K., 1994, ApJ 437,683

Puget J. L., Léger A., 1989, A \&AR 27, 161
Sandford S.A., Allamandola L.J., Tielens A.G.G.M., Valero L.J. 1988, ApJ 329, 498

Sandford S.A., Allamandola L.J., Tielens A.G.G.M., Sellgren K., Tapia M., Pendleton Y., 1991, ApJ 371, 607

Schmitt B., 1994, in: Molecules and grains in space, ed. Nenner I. AIP press, New York, 735

Schutte W. A., Greenberg J. M., 1991, A\&A 244, 190

Schutte W. A., 1996, in: The cosmic dust connection, ed. Greenberg J. M., in press

Smith R. G., Sellgren K., Tokunaga A. T., 1989, ApJ 344, 413

Szczepanski J., Roser D.,Personette W., Eyring M., Pellow R., Vala M., 1992, J.Phys.Chem. 96, 7876

Tegler S.C., Weintraub D.A., Rettig T.W., Pendleton Y.J., Whittet D.C.B., Kulesa C.A., 1995, ApJ 439, 279

Tielens A.G.G.M., Hagen W., 1982, A\&A 114, 245

Tielens A. G. G. M., 1991, in: Solid state Astrophysics, North Holland, eds. Bussoletti E., Strazulla G., 29

Tielens A. G. G. M., Tokunaga A. T., Geballe T. R., Baas F., 1991, ApJ 381,181

van de Bult C. E. P. M., Greenberg J. M., Whittet D. C. B., 1985, MNRAS, 214, 289

van Thiel E., Becker D., Pimentel G. C., 1957, J.Chem.Phys. 27, 486

Tso T. L., Lee E., 1985, J.Phys.Chem. 89, 1612

Weber P., Greenberg J. M., 1985, Nature 316, 403

Whittet D.C.B., Walker H.J., 1991, MNRAS 252, 63

Whittet D. C. B., Duley W. W., 1991, A\&AR 2, 167

Whittet D. C. B., 1993 in: Dust and chemistry in astronomy, eds. Millar T. J., Williams D. A., IOP Publ. Ltd. Bristol, 1

Willner S. P., Gillet F. C., Herter T. L., et al., 1982, ApJ 253, 174

This article was processed by the author using Springer-Verlag $\mathrm{IAT}_{\mathrm{E}} \mathrm{X}$ A\&A style file version 3 . 